\title{
Mit dem Tablet dem Formularwust entgehen
}

\author{
Erstanamnese, Aufklärungsbögen, Einverständniserklärung - in \\ der Praxis von Stefanie Baum und Dr. Sonja Ehlen füllen Patienten \\ solche Formulare nur noch auf dem Tablet aus. Das bringt den \\ Ärztinnen mehr Zeit für ihre Patienten und stärkt die Bindung an \\ die Praxis.
}

Die Idee kam Stefanie Baum, als sie hinten in ihrer Praxis ins Büro kam. Dort sah die Dermatologin einen Stapel mit Formularen, ,gefühlt einen Meter hoch“. Die Formulare füllten Patienten aus, wenn sie zum ersten Mal in die Praxis kamen.

„Wir waren mit dem Erfassen sechs bis acht Monate hinterher", erinnert sich die Hautärztin, die zusammen mit ihrer Kollegin Dr. Sonja Ehlen in der „Hautarztpraxis im vorderen Westen " in Kassel niedergelassen ist. Hinzu kam, dass bei der Übertragung in die EDV viele Fehler passierten. Die Formulare zur Erstanamnese, zu Recall-Einverständnis und ähnlichem mehr auf Papier waren den Ärztinnen daher schnell ebenso ein Dorn im Auge wie die vielen Aufklärungsformulare, die bei den auch operativ tätigen Dermatologinnen anfielen. Die Formulare machten viel Arbeit, noch dazu erzeugte die Praxis viel Papiermüll, die Bögen mussten alle durch den Shredder durch, wenn der Inhalt in der EDV gespeichert war.

Ein Aspekt war auch, dass der Aufwand der Ärztinnen mit der Verwaltung bei neuen Patienten in keinem Verhältnis stand zur reinen Behandlungszeit. „Häufig dauert ein kleiner Eingriff beim Patienten nur eine Minute, aber die Verwaltung mit der Aufklärung zieht sich über zehn Minuten“, erläutert Baum.

\section{Formulare, Formulare ...}

Es kam auch einiges zusammen an Formularen: ein Bogen für die Erstanamnese, Einverständniserklärungen für die Zusendung des Praxis-Newsletters, für die Erinnerung an einen Termin oder die anstehende Hautkrebsvorsorge, Aufklärungsbögen mit Unterschrift des Patienten vor einem Eingriff, Kostenaufklärung über Selbstzahlerleistungen - alles das füllten die Patienten geduldig auf Papier aus, das Praxisteam musste die Daten dann in die EDV händisch übertragen oder die Formulare einscannen.

Heute läuft es in der Praxis ganz anders - dank handlicher Tablet-Computer. Baum und Ehlen haben die Formulare in IT-Form überführt, Patienten füllen die Erstanamnesebögen jetzt im Wartezimmer aus und unterschreiben auch direkt auf dem Tablet ihre Einverständniserklärungen, wenn nötig. Die Software ist in die Praxis-EDV integriert. Speichert der Patient oder die Patientin ein Formular oder einen Anamnesebogen ab, wird das im System sofort sichtbar - „für jeden wichtigen Punkt gibt es eine Zeile in der elektronischen Kartei, so kann ich das mit einem Blick erfassen, ich muss nicht noch mal ins Dokument reingehen", beschreibt Baum die für sie optimierte Lösung.

Baum und Ehlen arbeiten mit Medistar. Der Vertriebspartner des Softwarehauses hat die Lösung für die Praxis erarbeitet und umgesetzt. „Wir haben die richtigen Leute gefragt, das ging auch nicht sofort, aber jetzt funktioniert es", berichtet die Dermatologin. Mit ihrer privaten Lösung zum Bürokratieabbau bewarben sich Baum und Ehlen beim Wettbewerb „Die innovative Arztpraxis 2014“, den das Biopharmaunternehmen UCB und die Fachverlagsgruppe Springer Medizin, zu der auch die „Ärzte Zeitung“ zählt, im vergangenen Jahr zum vierten Mal ausgeschrieben hatten.

Sie gewannen mit ihrem Beitrag zu effizienten Abläufen in der Praxis den ersten Preis. Wer auf die Website der beiden Hautärztinnen geht, wird als erstes von einem Bild empfangen, auf dem beide Ärztinnen die Trophäe in der Hand halten.

\section{"Patienten finden das super"}

Die Patienten fühlen sich von der Technik nicht überfahren, im Gegenteil: „Die finden das super", berichtet Baum, „wir bekommen fast ausschließlich positive Rückmeldungen." Denn es gibt noch einen positiven Nebeneffekt der Effizienzsteigerung: Wartezeit wird sinnvoll überbrückt, der Umgang mit der Software vermittelt auch etwas Spielerisches.

„Die jungen Leute sind sogar schlicht aus dem Häuschen." Von Informatikern hätten sie schon Angebote bekommen, neue Innovationen zu programmieren, etwa für Rezepte. „Die eine Idee regt an, weiter $\mathrm{zu}$ gehen, neue Ideen $\mathrm{zu}$ entwickeln", stellt Baum fest.

Die Tablets kommen überall zum Einsatz, wo es sinnvoll ist, im Wartezimmer, aber auch im Sprechzimmer. „Wenn wir bei der Hautkrebsvorsorge etwas entdecken und der Patient sich entscheidet, dass wir das gleich entfernen sollen, bekommt er direkt eine mündliche Aufklärung zu dem Eingriff und kann dann anschließend in Ruhe das Aufklärungsformular durchlesen und gleich auf dem TabletComputer unterschreiben", beschreibt Baum den üblichen Weg.

Für die Patienten ist dann mit einem Aufenthalt in der Praxis von der Vorsorge bis zum Eingriff gleich alles auf einmal erledigt. Die jungen Ärztinnen kommunizieren auch sonst gerne online, auch mit zuweisenden Kolleginnen und Kollegen allerdings nur so weit, wie es die Berufsordnung und der Datenschutz zulassen. Zum Beispiel beschrieben Zuweiser Symptome von nicht genannten Patienten und fragten, ob sie sie vorbeischicken sollen.

Eine Ferndiagnose, ohne den Patienten zu kennen, ist bekanntlich weiterhin verboten. Was möglich ist: „Ein von mir operierter Patient, der sich im Ausland aufhält, macht ein Foto von der Wunde und schickt es online zur Kontrolle zu." Hier geht es nicht um eine Behandlung ausschließlich per Datenleitung, und das ist erlaubt.

Baum berichtet, der Berufsverband der Dermatologen arbeite daran, den Spielraum bei Fernbehandlungen zu erweitern. Innovationen in diesem Feld hat es in den vergangenen Jahren bereits einige gegeben.

Hauke Gerlof 